\title{
Das papierlose Büro rechnet sich
}

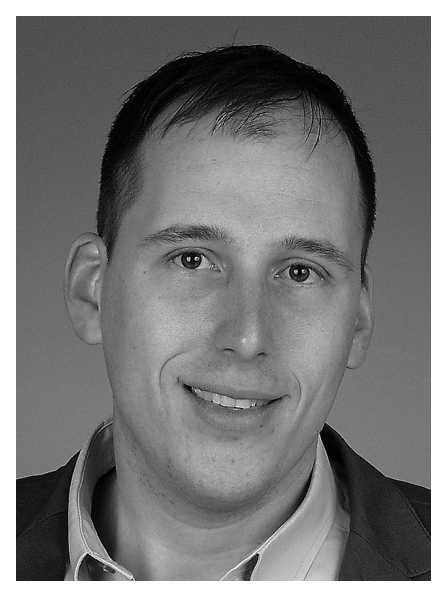

VON STEPHAN JOACHIM BÖCK Stephan Joachim Böck ist DiplomSozialpädagoge (FH) und seit 2003 als selbstständiger Berufsbetreuer in der Bürogemeinschaft Ottobeuren tätig. Seit 2011 ist er Landesvorstand und Finanzverantwortlicher der bayerischen Landesgruppe des Bundesverbandes der Berufsbetreuer/innen e. V. www.betreuungsbueroottobeuren.de

\author{
Durch die Investition in Informationstechnik und die \\ konsequente Anpassung der organisatorischen Abläufe \\ können sich auf Dauer positive betriebswirtschaftliche \\ Effekte ergeben, wie das Beispiel eines \\ Gemeinschaftsbüros von Berufsbetreuern zeigt.
}

Die Anforderungen an die Verwaltungen der Sozialwirtschaft sind in den vergangenen Jahren zunehmend gestiegen. Dokumentationspflichten in der Pflege und die bürokratischen Hürden bis zur Hilfegewährung in der Eingliederungshilfe sind nur zwei kleine Beispiele aus dem Alltag. Die Kosten für den hieraus resultierenden administrativen und in der Regel personalintensiven Aufwand steigen stetig an.

Eine Möglichkeit für Sozialunternehmen, dieser Fragestellung organisatorisch und betriebswirtschaftlich zu begegnen, wird hier am Beispiel des Betreuungsbüros Ottobeuren skizziert. Bei der Bürogemeinschaft handelt es sich um einen organisatorischen Zusammenschluss von zehn selbstständigen Einzelunternehmerinnen und Einzelunternehmer, die überwiegend Betreuungen

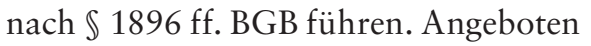
wird dies als Humandienstleistung, die neben einer anspruchsvollen beratenden, unterstützenden und vertretenden sozialarbeiterisch geprägten Tätigkeit, einen hohen bürokratischen Verwaltungsaufwand mit sich bringt. Das berufliche Betreuungswesen ist in der Regel in Form von selbstständigen Einzelunternehmern - als »Einzelkämpfer« oder Bürogemeinschaften - und Betreuungsvereinen, die sich oftmals in Trägerschaft von Wohlfahrtsverbänden befinden, geordnet.

Die Ausgangslage ist bei der Dienstleistung »Berufsbetreuung ", analog zu anderen kleineren sozialwirtschaftlich geprägten Anbietern, maßgeblich davon geprägt, dass aus den erzielten Umsätzen in der Regel kein personalintensiver Verwaltungsapparat finanziert werden kann. Um dem steigenden Verwaltungsaufwand ohne Aufblähung des Verwaltungsapparates zu begegnen, setzt das Betreuungsbüro Ottobeuren die Idee eines "papierlosen Büros « konsequent um. Um Missverständnissen vorzubeugen: Hierunter wird keine papierfreie Verwaltung verstanden, sondern vielmehr eine technikbasierte Büroorganisation. Diese beinhaltet als zentralen Baustein die vollständige Digitalisierung aller relevanten Dokumente und Nutzung elektronischer Zugriffs- und Versandwege.

Ein Betreuungsverfahren ist in der Regel mit einer aufwendigen und "papierlastigen « Aktenführung verbunden. Dokumente müssen häufig gelesen, bearbeitet, vervielfältigt und weitergeleitet werden. Der anfallende Zeit- und Kostenaufwand für die Führung, Nutzung und Lagerung der Akten stellt folglich einen Bürokostenfaktor dar. Durch die Nutzung neuer digitaler Techniken kann der Workflow effizient und kosteneinsparend gestaltet werden.

An erster Stelle steht naturgemäß die Datenerfassung. Eingehende Dokumente werden eingescannt und personenbezogen abgespeichert. Eine installierte Messaging-Software nimmt Faxe bereits digital als E-Fax entgegen, zeigt diese am jeweiligen Arbeitsplatz an und bietet unmittelbar Speichermöglichkeiten an. Zur späteren Dokumentenverwaltung erhalten Dateien logische und einheitliche Zuordnungsmerkmale. Für eine optimale Nutzung des Dateibestandes ist dringend der Einsatz einer professionellen Dokumentverwaltung zu empfohlen. Im Betreuungsbüro Ottobeuren 
umfasst der digitale Aktenbestand inzwischen rund 55.000 Dateien. Je nach den spezifisch geltenden Aufbewahrungspflichten können, eine zertifizierte Datensicherung natürlich vorausgesetzt, Dokumente in Papierform zur Reduzierung der Lagerkosten vernichtet werden.

Den entscheidenden und langfristig profitabelsten Anteil stellen die vielfältigen Möglichkeiten der Nutzung der digitalen Aktenbestände dar. Ein Zugriff auf die gespeicherten Informationen kann über jeden mit einer entsprechenden Freigabe versehenen Arbeitsplatz im Netzwerk erfolgen. Informationen wie Aktenzeichen, Telefonnummern, Ansprechpartner, Zahlen sind jederzeit problemlos und unkompliziert abrufbar. Duplikate lassen sich über die Nutzung von Einzelplatz- oder zentralen Netzwerkdruckern mühelos erstellen.

Die in der Regel an Verwaltungskräfte delegierten Arbeitsschritte - suchen, herausnehmen, aufklammern, kopieren, weiterleiten, zusammenhaften und wieder ablegen von Dokumenten - sind somit entweder überflüssig oder wesentlich effizienter durchführbar. Durch Nutzung elektronischer Versandwege via E-Mail oder E-Fax ist ein physischer Zugriff überhaupt nicht mehr nötig. Eingehende Faxe sind beispielsweise bereits am Bildschirm bearbeit- und durch den Einsatz digitaler Unterschriften ohne Ausdruck versendbar. Die häufigsten Engstellen im Büroalltag mit Warteschlagen vor dem Kopierer und Faxgerät sind folglich im Betreuungsbüro Ottobeuren nachhaltig beseitigt. Die im Umfang deutlich reduzierte »Papierakte " wird tatsächlich nur noch in Ausnahmefällen zur Hand genommen.

Sehr flexibel lässt sich ein Fernzugriff auf das Netzwerk nutzen. Über einen eingerichteten Remote-Server ist, eine ausreichende Datenverbindung vorausgesetzt, jederzeit und von jedem Ort aus ein Einloggen in das Netzwerk über internetfähige Endgeräte (PC, Laptop, Notebook, Tablet, Smartphone etc.) möglich. Somit lässt sich mobil auf alle Daten zugreifen und eine branchenspezifische netzwerkfähige Software abseits des Arbeitsplatzes nutzen. In Kombination mit einem Messaging Programm entsteht somit ein vollwertiger Arbeitsplatz, der beispielsweise via Tablet selbst auf einer Parkbank genutzt werden kann. Gerade im Dienstleistungssektor wird hierdurch ein Mehr an Unabhängigkeit, Flexibilität und Kundenorientierung ermöglicht.

\section{Gute Gründe für eine Reorganisation}

Die spannende Frage ist natürlich: Für wen lohnt sich die Investition in eine papierlose Verwaltung und welcher Aufwand ist hiermit verbunden? Die erste Fragestellung kann erfahrungsgemäß mit Ja beantwortet werden, wenn monatlich hohe Kopier- und Portokosten anfallen, Dokumente beständig vervielfältigt und weitergeleitet werden, häufig Akten herangezogen und aufbewahrt werden, eine umfangreiche schriftliche Korrespondenz erforderlich ist, papierlastige Vorgänge bearbeitet werden, Daten und Informationen erfasst und regelmäßig abgerufen werden.

Diese Punkte sind von besonderer Relevanz, falls hierfür persönliche Arbeitszeit aufgewendet oder Personal für diese Tätigkeiten eingesetzt wird. Ist die Entscheidung für die »papierlose Verwaltung « getroffen, steht zunächst die Schaffung einer geeigneten technischen Infrastruktur an. Als in der Praxis gut einsetzbar hat sich im Betreuungsbüro Ottobeuren eine Kombination aus mehreren Komponenten erwiesen. Diese besteht aus einem Windows Small Business SQLServer, der Messaging-Software »David. Fx« von Tobit für E-Mail und E-Fax, einer netzwerkfähigen branchenspezifischen Software, der Adobe Acrobat Standard Version zur Bearbeitung von PDF-Dokumenten, einem Netzwerkdrucker mit Festplatte und Netzwerk- und Einzelplatzdokumentenscannern mit einer leistungsfähigen Scan- und Verwaltungssoftware. Bei Letzteren sollte auf eine hohe Scanleistung (Seiten pro Minute) und »Duplexfunktion« (gleichzeitiges Erfassen von Vorder- und Rückseite) geachtet werden. Die hierfür anfallenden Kosten belaufen sich wie folgt (Überschlag für fünf Arbeitsplätze):

- Messaging Software ca. 850,- Euro

- SQL-Server ca. 2.500,- Euro

- Dokumentenscanner ab 800,- Euro

- Installationskosten ca. 1.000,- Euro

- Datensicherung, Wartung, Updates, Virenscanner, Firewall etc. ab 500,Euro jährlich

Neben der Installation einer geeigneten technischen Basis muss die Büroorga- nisation hierfür umstrukturiert und folgende Fragestellungen bürointern abgestimmt werden:

- Wie wird die Idee einer papierlosen Verwaltung in den Arbeitsablauf integriert? Welche Dokumente werden wann und von wem erfasst, eingelesen und abgespeichert?

- Wie sieht die Nutzung der digitalen Datenbestände aus? Wer erhält welche Zugriffsrechte? In welcher Struktur werden die Dateien abgespeichert? Wie sieht die Dateiverwaltung aus? In welcher Form sollen die digitalen Dokumente später verwendet werden?

- Was geschieht mit den eingescannten Originalen in Papierform? Bestehen Aufbewahrungspflichten oder können diese zur Vermeidung von Archivierungskosten entsorgt werden?

Aufgrund der technischen Komplexität ist es ratsam, für die fortlaufende Betreuung einen externen IT-Dienstleister $\mathrm{zu}$ beauftragen und gegebenenfalls einen Wartungsvertrag abzuschließen. Aus datenschutzrechtlichen Gründen muss eine ausreichende Absicherung des Netzwerkes gegen unbefugte $\mathrm{Zu}$ griffe von außen - Viren, Trojaner etc. - erfolgen. Auch bedarf es einer zuverlässigen und regelmäßigen Datensicherung. Der Nutzwert einer digitalen Aktenstruktur erhöht sich im Laufe der Zeit durch den zunehmenden Umfang der digitalisierten Dokumente. Der hierin enthaltene Zeitaufwand stellte einen Wert dar, den es verlässlich gegen Datenverlust zu sichern gilt.

\section{Resümee}

Durch die effiziente Bearbeitung der Verwaltungsaufgaben konnten im Betreuungsbüro Ottobeuren zeitliche Freiräume für das Kerngeschäft, der persönliche Kontakt mit Menschen in komplexen Problemlagen, geschaffen werden. Auch hat sich das bürointerne Anforderungsprofil für Bürokräfte gewandelt. Da keine zeitaufwendigen Zuarbeiten wie Kopieren mehr erforderlich sind, steht unseren Mitarbeitenden mehr Zeit für komplexere und den Sozialunternehmer entlastende Unterstützungstätigkeiten zur Verfügung. Die mobilen Zugriffsmöglichkeiten auf das Netzwerk haben zudem Freiräume zur flexiblen Nutzung der Arbeitszeit eröffnet. 